\title{
Reentrant and Isostructural Transitions in a Cluster-Crystal Former
}

\author{
Kai Zhang, ${ }^{1}$ Patrick Charbonneau, $, 1,2$, * and Bianca M. Mladek ${ }^{3, \text { † }}$ \\ ${ }^{1}$ Department of Chemistry, Duke University, Durham, North Carolina, 27708, USA \\ ${ }^{2}$ Department of Physics, Duke University, Durham, North Carolina, 27r08, USA \\ ${ }^{3}$ Department of Chemistry, University of Cambridge, Lensfield Road, CB2 1EW, UK
}

(Dated: September 20, 2018)

\begin{abstract}
We study the low-temperature behavior of a simple cluster-crystal forming system through simulation. We find the phase diagram to be hybrid between the Gaussian core model and the penetrable sphere model. The system additionally exhibits S-shaped doubly reentrant phase sequences as well as critical isostructural transitions between crystals of different average lattice site occupancy. Due to the possible annihilation of lattice sites and accompanying clustering, the system moreover shows an unusual softening upon compression.
\end{abstract}

PACS numbers: 64.70.K-,64.70.D-,82.30.Nr,62.20.-x

Since van der Waals and Kirkwood, we better appreciate the fundamental role of harshly repulsive interactions in the organization of matter [1]. But what happens when harshness turns into softness? Core softened potentials can exhibit microphase separation 2], reentrant melting, and isostructural phase transitions [3], as found in systems as diverse as Cerium metal [4], star polymers [5], dipolar spheres [6], electron bubbles [7, [8], and rotating Bose gases [9]. Even softer coreless repulsive interactions are also found in complex systems. Nonlinear fields can form particle-like structures governed by soliton-like interactions [10, 11]; and for structures with low fractal dimension, such as polymers [12], dendrimers [13, 14], and microgels [15], the centers of mass can be immaterial and thus overlap with only a finite free energy penalty. As a result, materials governed by such interactions exhibit unusual phenomenology compared to "simple" matter [16]. Soft core models are further used to study the difficult glass [17, 18] and classical ground state determination problems [19, 20], highlighting the broad interest in the behavior of systems with soft potentials.

A certain universality permeates the thermodynamic assembly of systems governed by bounded, purely repulsive interactions. Two phenomenological categories have been identified from a mean-field analysis. Systems with pair interactions whose Fourier components are purely positive are expected to show reentrant melting, while those with some negative Fourier components should cluster and freeze into multiply occupied crystals (MOC) upon compression [17, 21 27]. Crossing the divide between the two categories can be realized via the tuneable generalized exponential model of index $n$ $($ GEM- $n$ ) [23], whose pair potential for particles a distance $r$ apart is

$$
\phi(r)=\varepsilon \exp \left[-(r / \sigma)^{n}\right],
$$

with $\varepsilon$ and $\sigma$ setting the units of energy and length, respectively [28]. Upon compression at low temperatures, the $n=2$ Gaussian core model (GCM) 29], which has a purely positive Fourier transform, forms cubic crystals that eventually remelt [30, 31]; while all GEM- $n$ of $n>2$ have some negative Fourier components and are thus predicted to form MOC at high temperatures 22], including the $n \rightarrow \infty$ penetrable sphere model (PSM) limit [21].

For strong interactions (or effective low temperatures), which may be the experimentally most relevant regime, the phase behavior of MOC-forming systems is not understood. Various plausible ordering scenarios upon compression are suggested by theory and experiments: density functional theory predicts a continuous increase in clustering 22, 23]; the PSM limit presents a sequence of second-order phase transitions between crystals of increasing occupancy [21]; and bubble solids alternate liquid and crystal phases of increasing lattice occupancy [7]. A cascade of pure first-order isostructural solid-solid transitions between crystals of different occupancy is also plausible, as theoretically predicted by Ref. [32]. Such isostructural transitions should terminate at critical points, because the cluster occupancy of hightemperature MOC-formers increases continuously with density [23, 24]. A rare example of this type of critical point is found in pure Cerium, where a pressureinduced electronic promotion underlies the transition between two isostructural solids with different lattice spacing [4, 33]. Critical points involving volume collapse have also been predicted for a variety of purely classical, discontinuous model potentials [3, 34]. But the experimental colloidal systems in which they could be observed have intrinsic size polydispersity [35] or smooth effective interactions [16] that inhibit phase separation. The relatively broad lattice spacing of MOC and their smooth interaction form suggest that crystal formation should be less sensitive to these experimental constraints.

In this letter, we present a computational study of the low-temperature behavior of the MOC-forming GEM-4. Its phase behavior is shown to be a complex hybrid between the GCM and the PSM limits. We furthermore find complex reentrant transitions and evidence for a cascade of isostructural transitions.

We perform lattice Monte Carlo simulations [36] of 

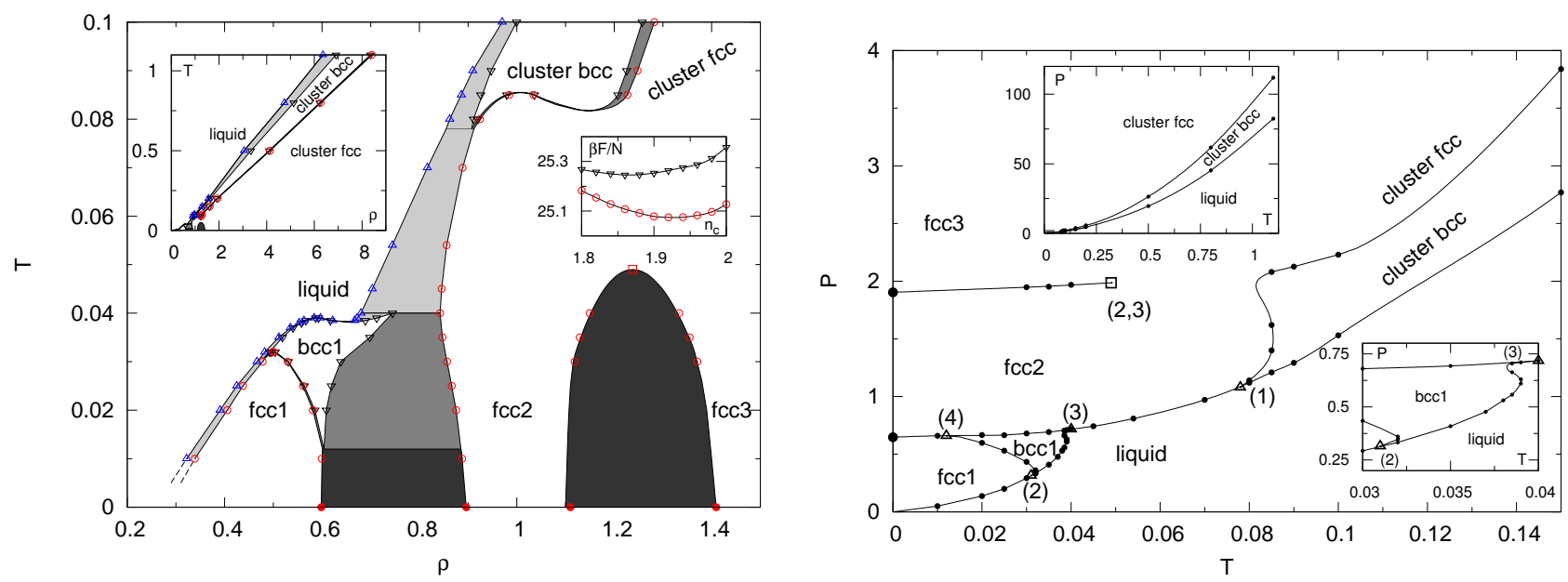

FIG. 1: (Color online) $T$ - $\rho$ (left) and $P-T$ (right) low- and high- $T$ (left insets 24]) simulation phase diagrams. The isostructural critical point $(\square)$ is extrapolated from the law of rectilinear diameters [37] and the $T=0$ results $(\bullet)$ come from phonon theory [32]. Left: The coexistence regions (shaded) are delimited by simulation results for liquid $(\Delta)$, bcc $(\nabla)$, and fcc $(\odot)$ phases. The free energy per particle for the bcc $(\nabla)$ and fcc $(\odot)$ phases at $T=0.03$ and $\rho=0.85$ shows that a fcc with $n_{c}^{\text {eq }}=1.94(1)$ is the ground state (right inset). Right: The phase boundaries (solid lines) are guides for the eye that are consistent with the Gibbs-Duhem slopes (not shown) at the coexistence points $(\cdot)$. The triple points $(\Delta)$ are numbered. The right inset enlarges the SDR liquid-bcc1-liquid-bcc1 sequence and the bcc1-fcc1-bcc1 crystal reentrance.

the GEM-4 model, whose high-temperature behavior was previously determined [24], for $N=1000-5000$ particles at constant $N$, volume $V$, and temperature $T$. The pressure $P$ is obtained from the virial and the Helmholtz free energy $F$ of the different phases is calculated via thermodynamic integration. The ideal gas reference 37. is complemented, for the body-centered cubic (bcc) and face-centered cubic (fcc) crystal phases, with a potential well centered around each of the $N_{c}$ lattice sites [27]. This crystal reference, by allowing for the characteristic multiple occupation of lattice sites and for particle hopping between those sites, permits a reversible integration path. For a fixed number density $\rho$, the average lattice site occupancy $n_{c}=N / N_{c}$ at equilibrium is identified for every state point by simulating crystals at various fixed $n_{c}$ then minimizing the resulting constrained free energy $F\left(n_{c}\right)$, i.e., identifying the loci $F\left(n_{c}^{\text {eq }}\right)$ such that (Fig. 1)

$$
\left[\frac{\partial F\left(n_{c}\right)}{\partial n_{c}}\right]_{\rho, T, n_{c}=n_{c}^{\mathrm{eq}}}=0
$$

similarly to the scheme employed in Ref. [38] and that used for GEM-4's high- $T$ phase diagram determination. In the latter, $n_{c}$ was tuned until the (unphysical) field conjugate to $n_{c}$ had vanished [24, 39], which allows for a gradient-based minimization of $F$, but relies on an additional independent calculation of the chemical potential $\mu$. This approach breaks down at low $T$, where $\mu$ cannot be efficiently resolved by Widom's particle insertion [37]. Phase coexistence is then determined through common tangent construction of the free energy data. A linear transformation $\beta \tilde{F} \rho / N=\beta F \rho / N-\kappa \rho$ with inverse temperature $\beta$ and arbitrary unitless parameter $\kappa$ enhances the visibility of the coexistence regime (Fig. 2).

We present two projections of the the low- $T$ phase diagram in Fig. 1. As anticipated from the high- $T$ extrapolation, the cluster bcc phase vanishes at a triple point $T_{\mathrm{t}}^{(1)}=0.078(1)$. But surprisingly the transition is preceded by a S-shaped doubly reentrant (SDR) crystal phase sequence, where both a maximum and a minimum in the coexistence curve are observed. Below $T_{\mathrm{t}}^{(1)}$ the phase diagram is surprisingly rich. GCM-like phase behavior 29 31] is followed by an unexpectedly complex clustering regime. Because the tail of the GEM-4 decays faster than any inverse power, the liquid freezes into a single-occupancy fcc (fcc1) that reaches vanishingly small densities at low $T$, in agreement with predictions from genetic algorithms [40, 41] and phonon theory [32]. This fcc1 phase gives way to a single-occupancy bcc (bcc1) phase at a second triple point $T_{\mathrm{t}}^{(2)}=0.031(1)$. For a narrow temperature range above $T_{\mathrm{t}}^{(2)}$, a bcc1 wedge between the liquid and the fcc 1 phase leads to reentrant crystallization of bcc1 upon compression. The maximum freezing temperature for bcc1 at $T=0.039(1)$ leads to a second SDR sequence similar to that observed in the hard core/soft shoulder model [3, 4]. By contrast to the GCM's reentrant melting behavior [29 31], here the liquid reentrance section of the SDR sequence spreads only over a finite density regime $0.59 \lesssim \rho \lesssim 0.68$ and over a much smaller temperature range $0.0385 \lesssim T \lesssim 0.039$. The intermediate nature of the GEM- 4 suggests that this reentrance might become more pronounced as the GCM is approached, i.e., $n \rightarrow 2^{+}$, and should disappear before the PSM limit $n \rightarrow \infty$, where reentrance is not expected 21]. The connection between the high 


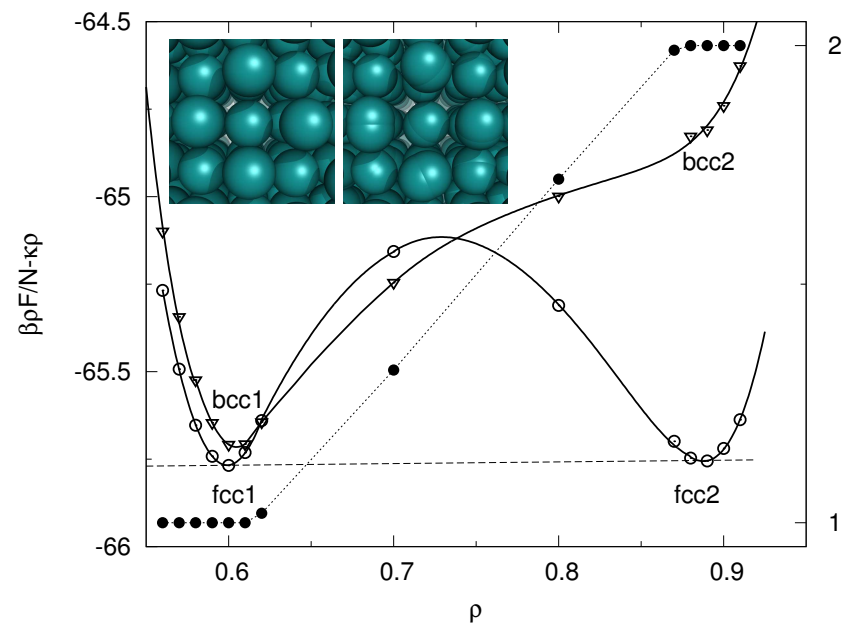

FIG. 2: Free energy curves of the stable fcc $(\odot)$ and, for comparison, the metastable bcc $(\nabla)$ structures at $T=0.01$ with $\kappa=142.7$. Both curves show the van der Waals loop characteristic of a system whose limited size inhibits phase separation. The coexistence densities of the fcc1 (left inset)fcc2 (right inset) isostructrual transition are determined by the common tangent construction (dashed line). The equilibrium lattice occupancy $n_{c}^{\mathrm{eq}}(\bullet)$ plateaus near integer values for the thermodynamically stable phases.

and low- $T$ regimes occurs through a third triple point $T_{\mathrm{t}}^{(3)}=0.040(1)$, at which bcc1 vanishes. A prior, coarser study of the liquid-crystal transition in this regime missed both the presence of fcc1 and of the reentrant melting [42]. It also inaccurately assigned the unusual shape of the liquid-crystal coexistence curve to the onset of clustering, while it is rather caused by the reentrant melting.

Clustering does indeed influence the phase diagram topology, but at densities further away from the liquid phase. At low $T$ the nature of clustering is unlike what is seen at higher $T$, where $n_{c}$ changes linearly with $\rho$ resulting in a nearly density independent lattice constant 24$]$. Here, $n_{c}$ is quasi-quantized, and at very low $T$ the lattice constant $a$ changes discontinuously through isostructural transitions between fcc lattices of nearly perfect integer mean occupancy $n_{c} \leftrightarrow n_{c}+1$ (Figs. 2] 3). The first occurrence of these transitions, fcc1 $\leftrightarrow$ fcc 2 , is partially interrupted by the bcc1 phase, down to the fourth triple point $T_{\mathrm{t}}^{(4)}=0.012(1)$ (Fig. 11). But at higher densities, the fcc $2 \leftrightarrow$ fcc 3 coexistence is fully developed. No other liquid or crystal phases are found to interfere and genetic algorithm results corroborate that no other crystal symmetry should be stable in this density regime [40, 41]. It is at the moment computationally difficult to go beyond fcc3, but both a zero temperature treatment paired with phonon theory 32] and a simple mean-field cell theory predict a cascade of $n_{c} \leftrightarrow n_{c}+1$ isostructural transitions to carry on ad infinitum, slightly broadening the coexistence regime between two integer occupancies. As argued above, the topology of the phase diagram

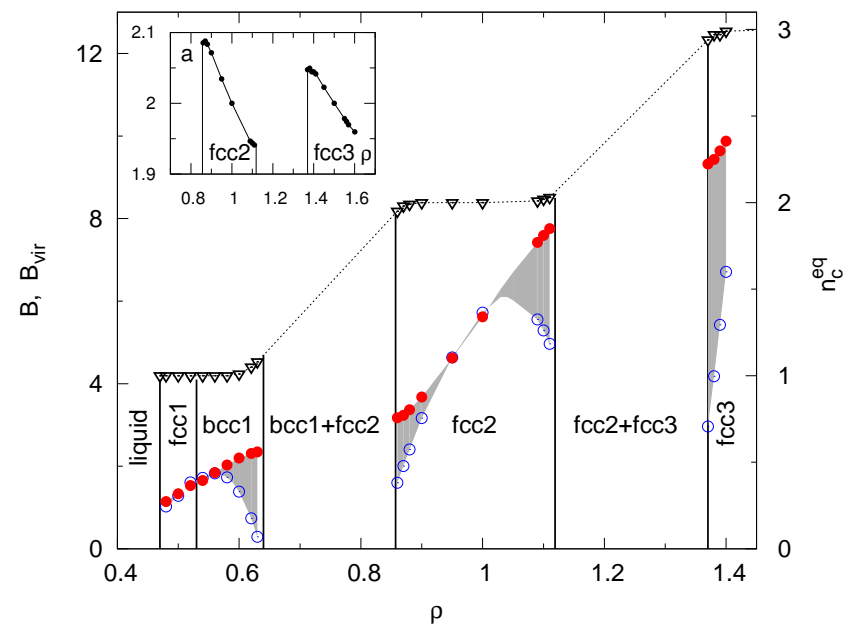

FIG. 3: (Color online) Isothermal $(T=0.03)$ bulk modulus $B(\odot)$, its virial contribution $(\bullet)$ and softening correction (shaded), and $n_{c}^{\mathrm{eq}}(\nabla)$ [45]. The solid vertical lines indicate phase boundaries. The lattice constant $a$ changes discontinuously as fcc $2 \rightarrow$ fcc3 (inset).

demands that each uninterrupted isotructural transition terminates at a critical point, the first one of which is found at $T_{c}^{(2,3)}=0.049(3)$. Hopping between lattice sites should depress $T_{c}^{\left(n_{c}, n_{c}+1\right)}$ as $n_{c}$ increases, and mean-field critical universality is generally expected [22, 43]. The series of first-order isostructural transitions contrasts with the continuous second-order clustering transitions for the PSM predicted by cell theory [21]. This last observation suggests that the behavior of the PSM might be singular, but further studies are necessary to clarify the GEM- $n$ family phase behavior as $n \rightarrow \infty$.

One of the key material properties of MOC is the presence of two distinct microscopic mechanisms for responding to compression. Like any other crystal, MOC can affinely reduce their lattice constant, but additionally they can eliminate lattice sites by increasing the mean lattice occupancy. We can decompose the bulk modulus

$$
B \equiv V\left(\frac{\partial^{2} F}{\partial V^{2}}\right)_{N, T}=B_{\mathrm{vir}}-B_{\text {corr }}
$$

into a constant $n_{c}$ virial contribution $B_{\text {vir }}$ and a "softening" correction $B_{\text {corr }}$, which map directly to the two microscopic mechanisms [24, 44]. At high $T, B_{\text {corr }}$ can be as high as half the virial component [24], but in low $T$ crystals, the quasi-quantized jumps in $n_{c}$ lead to a significantly different mechanical behavior. Away from the coexistence regions, where $n_{c}$ is nearly constant, the system responds only affinely to isothermal compression and the virial contribution to the bulk modulus captures the full response of the system, i.e., $B_{\text {corr }} \sim 0$, (Fig. 3) 45). But in the softening regions that precede and follow the phase transitions the quantization is imperfect, and $B_{\text {corr }} \neq 0$. 
Near the bcc1-fcc2 transition, for instance, $B_{\text {corr }}$ is nearly equal to the virial contribution, which means that the system exerts almost no resistance to compression. This very rapid change in mechanical properties with compression is uncommon, and may lead to novel material behavior. The different physical natures of the virial and softening contributions indeed suggest a separation of time scales for their microscopic relaxation, with slow particle redistributions contrasted by fast affine deformations. Hardening or softening of the material upon compression might thus depend on the deformation rate.

We have presented the intriguing low-temperature phase behavior of the MOC-forming GEM-4 through a numerical method specially designed for this class of systems. The complexity of the phase behavior is particularly noteworthy considering the simplicity of the model, which is free of competing length scales. Experimental soft matter realizations of MOC are still lacking, but large-scale, monomer-resolved simulations of amphiphilic cluster-forming dendrimers are currently under way [46]. Importantly, the approach outlined in the present work should be directly applicable to phenomena of reversible cluster formation in other branches of physics. Examples are: the structures formed by the soft solitons [10, 11], the quasi-2D electron bubbles in the quantum-Hall regime [7, 8], and the predicted clustering of vortex lines in rotating Bose gases [9].

We thank D. Frenkel, O. Poplavskyy, N. Cooper (Cambridge), and C. N. Likos (Vienna) for helpful discussions, and A. Dawid (Grenoble) for careful reading of the manuscript. $\mathrm{KZ}$ and $\mathrm{PC}$ acknowledge ORAU and Duke startup funding. BMM acknowledges EU funding via FP7-PEOPLE-IEF-2008 No. 236663.

* Electronic address: patrick.charbonneau@duke.edu

$\dagger$ Electronic address: bmm32@cam.ac.uk

[1] J. D. Van der Waals, On the Continuity of the Gaseous and Liquid States (Elsevier Science Publishers, New York, 1988).

[2] M. A. Glaser et al., Europhys. Lett. 78, 46004 (2007).

[3] D. A. Young and B. J. Alder, J. Chem. Phys. 70, 473 (1979).

[4] D. A. Young, Phase Diagrams of the Elements (University of California Press, Berkeley, 1991).

[5] M. Watzlawek, C. N. Likos, and H. Löwen, Phys. Rev. Lett. 82, 5289 (1999).

[6] N. Osterman, D. Babic, I. Poberaj, J. Dobnikar, and P. Ziherl, Phys. Rev. Lett. 99, 248301 (2007).

[7] M. O. Goerbig, P. Lederer, and C. M. Smith, Phys. Rev. B 68, 241302(R) (2003).

[8] O. Poplavskyy, M. O. Goerbig, and C. Morais Smith, Phys. Rev. B 80, 195414 (2009).

[9] N. R. Cooper, Adv. Phys. 57, 539 (2008).

[10] I. S. Aranson, K. A. Gorshkov, A. S. Lomov, and M. I. Rabinovich, Physica D 43, 435 (1990).
[11] A. V. Gomez, P. Alstrøm, and S. Toxvaerd, Phys. Rev. B 51, 2822 (1995).

[12] A. A. Louis, P. G. Bolhuis, J.-P. Hansen, and E. J. Meijer, Phys. Rev. Lett. 85, 2522 (2000).

[13] B. M. Mladek, G. Kahl, and C. N. Likos, Phys. Rev. Lett. 100, 028301 (2008).

[14] C. N. Likos et al., J. Chem. Phys. 117, 1869 (2002).

[15] D. Gottwald, G. Kahl, and C. N. Likos, J. Chem. Phys. 122, 204503 (2005).

[16] C. N. Likos, Phys. Rep. 348, 267 (2001).

[17] W. Klein, H. Gould, R. A. Ramos, I. Clejan, and A. I. Mel'cuk, Physica A 205, 738 (1994).

[18] A. Ikeda and K. Miyazaki (2010), arXiv:1008.2597.

[19] S. Torquato and F. H. Stillinger, Phys. Rev. Lett. 100, 020602 (2008).

[20] C. N. Likos, Nature 440, 433 (2006).

[21] C. N. Likos, M. Watzlawek, and H. Löwen, Phys. Rev. E 58, 3135 (1998).

[22] C. N. Likos, A. Lang, M. Watzlawek, and H. Löwen, Phys. Rev. E 63, 031206 (2001).

[23] B. M. Mladek, D. Gottwald, G. Kahl, M. Neumann, and C. N. Likos, Phys. Rev. Lett. 96, 045701 (2006).

[24] B. M. Mladek, P. Charbonneau, and D. Frenkel, Phys. Rev. Lett. 99, 235702 (2007).

[25] E. Lascaris, G. Malescio, S. V. Buldyrev, and H. E. Stanley, Phys. Rev. E 81, 031201 (2010).

[26] P. Ziherl and R. D. Kamien (2010), arXiv:1009.5288.

[27] B. M. Mladek, P. Charbonneau, C. N. Likos, D. Frenkel, and G. Kahl, J. Phys.: Cond. Matter 20, 494245 (2008).

[28] All quantities are reported in reduced units [37].

[29] F. H. Stillinger, J. Chem. Phys. 65, 3968 (1976).

[30] A. Lang, C. N. Likos, M. Watzlawek, and H. Löwen, J. Phys.: Cond. Matter 12, 5087 (2000).

[31] S. Prestipino, F. Saija, and P. V. Giaquinta, Phys. Rev. E 71, 050102 (2005).

[32] T. Neuhaus and C. N. Likos, J. Phys.: Condens. Matter (in press) (2010), arXiv:1008.1881.

[33] M. J. Lipp et al., Phys. Rev. Lett. 101, 165703 (2008).

[34] P. Bolhuis and D. Frenkel, J. Phys.: Cond. Matter 9, 381 (1997).

[35] S. Buzzaccaro, R. Rusconi, and R. Piazza, Phys. Rev. Lett. 99, 098301 (2007).

[36] A. Z. Panagiotopoulos, J. Chem. Phys. 112, 7132 (2000).

[37] D. Frenkel and B. Smit, Understanding Molecular Simulation (Academic Press, 2002).

[38] K. Zhang and P. Charbonneau, Phys. Rev. Lett. 104, 195703 (2010).

[39] W. C. Swope and H. C. Andersen, Phys. Rev. A 46, 4539 (1992).

[40] B. M. Mladek, D. Gottwald, G. Kahl, M. Neumann, and C. N. Likos, J. Phys. Chem. B 111, 12799 (2007).

[41] B. M. Mladek, Ph.D. thesis (2007).

[42] H. Fragner, Phys. Rev. E 75, 061402 (2007).

[43] T. Chou and D. R. Nelson, Phys. Rev. E 53, 2560 (1996).

[44] J. Rowlinson, Liquids and liquid mixtures (Butterworths Scientific Publications, London, 1959).

[45] The bulk modulus is obtained by differentiating empirical fits to the free energy results. Though this approach captures the mechanical behavior rather well, some numerical errors remain since the softening correction must clearly be positive.

[46] D. A. Lenz et al., J. Phys. Chem. B (in press) (2010). 